\title{
Parents and Pediatric Weight Management Attrition: Experiences and Recommendations
}

\author{
Bethany J. Sallinen Gaffka, PhD, Maura Frank, MD,2 Sarah Hampl, MD, ${ }^{3}$ \\ Melissa Santos, $\mathrm{PhD}^{4}$ and Erinn T. Rhodes, MD, $\mathrm{MPH}^{5}$
}

\section{Abstract}

Background: One of the most frequently cited challenges faced by pediatric weight management programs/clinics is attrition, with many studies reporting rates greater than $50 \%$. Few studies have evaluated parental perspectives on recommendations for weightmanagement treatment enhancement. The aim of this study was to elicit perspectives on areas for improvement, discussions with staff about discontinuation, and potentially modifiable aspects of attrition from parents who prematurely discontinued stage 3 pediatric weight management treatment.

Methods: This study was performed as a semistructured interview as part of a telephone survey assessing reasons for attrition.

Results: Interviews were performed with 147 parents of children who attended programs/clinics at 13 children's hospitals participating in the National Association of Children's Hospitals and Related Institutions (now Children's Hospital Association) FOCUS on a Fitter Future II collaborative. The majority of parents (65\%) denied talking to staff about their decisions to stop coming. When describing what could have been done to retain families, parents most frequently discussed changing logistics (e.g., hours and locations). Parents described changes in logistics and components (i.e., nutrition education, exercise, and behavior education/ support) when asked what would work best for their family for pediatric weight management.

Conclusions: Parental responses appeared to express frustration about flexibility with appointment times and treatment locations. The most frequently desired components were those traditionally offered by stage 3 pediatric weight management programs/clinics, and this may suggest a need for treatment delivery of these components to be more individualized. Additional discussion with families about their desire to discontinue treatment may provide a timely opportunity to address this need.

\section{Introduction}

$O$ eventeen percent of children and adolescents in the United States were obese and 32\% either overweight or obese in the years 2009-2010. ${ }^{1}$ This represents a tripling in rates of obesity over the last three decades. ${ }^{2}$ Childhood obesity is currently a recognized risk factor for a number of chronic conditions previously found only in adults. ${ }^{3-7}$ Additionally, obesity in children tends to track into adulthood, increasing the risk of these chronic diseases over the life cycle. ${ }^{8,9}$ These facts highlight the importance of both treatment and prevention strategies to reverse this trend.
Recent response to this crisis has been heartening. Multiple initiatives have been developed, both locally and nationally, to address childhood obesity. Nationally, First Lady Michelle Obama has made childhood obesity prevention a priority in her Let's Move! campaign. ${ }^{10}$ Children's hospitals nationwide have responded by developing weight management programs/clinics and conducting clinical trials. The need for these offerings is both supported and encouraged by a 2007 summary report of an expert committee's recommendations regarding childhood obesity. ${ }^{11}$ This report promotes a step-wise approach, consisting of four progressively intensive stages of

\footnotetext{
'Pediatric Comprehensive Weight Management Center, Department of Pediatrics, Division of Child Behavioral Health, University of Michigan C.S. Mott Children's and Von Voigtlander Women's Hospital, Ann Arbor, MI.

${ }^{2}$ Division of Academic General Pediatrics, New York-Presbyterian Phyllis and David Komansky Center for Children's Health, New York, NY. ${ }^{3}$ Weight Management and General Pediatrics, Children's Mercy Hospitals and Clinics and Center for Children's Healthy Lifestyles and Nutrition, Kansas City, MO.

${ }^{4}$ Division of Endocrinology, Connecticut Children's Medical Center, Hartford, CT.

${ }^{5}$ Division of Endocrinology, Boston Children's Hospital, Boston, MA.
} 
intervention. In stage 3, known as Comprehensive Multidisciplinary Intervention, children are referred for frequent, often weekly, visits with a team of obesity specialists, and stage 4 is stage 3 treatment with the addition of bariatric surgery, weight-loss medication, and/or a very-low-calorie diet. ${ }^{11}$ Children's hospitals offering stage 3 treatment have included office-based individual interventions, group sessions, and combinations of both. ${ }^{12,13}$ Some include the child and parents/caregivers, whereas others have targeted the parents/caregivers (hereinafter referred to as parents) alone. ${ }^{14}$ Many interventions include the services of pediatricians, dietitians, mental health professionals, and physical therapists and/or trainers. ${ }^{13}$

Although a number of reports demonstrate successful outcomes for such interventions, ${ }^{15,16}$ one of the most frequently cited challenges faced by pediatric weightmanagement programs/clinics is attrition. Rates of attrition have been reported between $27 \%$ and $73 \%$, with most studies reporting rates greater than $50 \% .{ }^{17}$ Attrition prevents a child from benefitting from a potentially effective intervention while also decreasing the intervention's effectiveness and cost-effectiveness. ${ }^{18}$ Therefore, determining predictors for dropout and clarifying the needs of children and parents regarding weight management are crucial to the success of these initiatives.

Few studies have focused on reasons for attrition from pediatric weight management. Predictive factors for dropout cited in these studies have been variable. Some studies correlate increased BMI, older age, African-American race/ ethnicity, nonwhite, non-Hispanic race/ethnicity, singleparent households, behavioral issues, poor health, and Medicaid insurance status with increased risk of attrition, but these findings have not been consistent. ${ }^{16-21}$ Though several qualitative studies focus on issues that affect success with pediatric weight management, ${ }^{22-24}$ few studies have specifically evaluated parental perspectives on contributors to attrition. ${ }^{18,25-29}$ In the few studies published that report on reasons parents give for dropout, loss of school time, location of the program, scheduling problems, insurance issues/cost of program, child's desire to leave the program, problems with program content, and perceived quality of care are cited. ${ }^{17,18,25}$ Parents in the study by Kitscha and colleagues recommend a family-centered approach, greater emphasis on physical activity, and ageappropriate information for children, ${ }^{27}$ whereas parents in the Cote and colleagues study recommend assistance with insurance problems. ${ }^{18}$ Parents and children in the Banks and colleagues study suggested an age-appropriate clinic environment, involvement of children in the decision to attend, and tailoring advice to the family. ${ }^{28}$ Similarly, families in the Visram and colleagues study noted that they appreciated tailoring of advice, but also recommended involving families in decision making and improved communication with staff. ${ }^{29}$

In 2008, the National Association of Children's Hospitals and Related Institutions (NACHRI), now the Chil- dren's Hospital Association, convened a work group on childhood obesity treatment (FOCUS on a Fitter Future I) and brought together representatives from 16 geographically diverse children's hospitals with established or emerging obesity treatment programs/clinics to address critical issues in this field. In FOCUS on a Fitter Future I (2008-2010), member hospitals identified attrition as a major barrier to the success of pediatric weight management initiatives. ${ }^{12}$ The second focus group (FOCUS on a Fitter Future II, 2010 2011) sought to investigate the reasons for attrition from the direct perspective of parents. Given the scant published data on parental perspectives on the critical features needed in pediatric weight management programs/clinics and their suggestions for improvement, the primary aim of this study was to elicit these perceptions from parents and search for common themes that could address the potentially modifiable contributors to attrition, development of new treatment initiatives, and improvement of existing ones to maximize family participation.

\section{Methods}

\section{Study Design}

A cross-sectional design was used to examine the perspectives of parents whose children discontinued stage 3 pediatric weight management treatment either in a clinic or program setting. For this study, "programs" were considered treatments that included a structured number of visits, and "clinics" were considered those that did not have a defined end point. Both treatment options could include individual and/or group visits, and some sites offered more than one option. Semistructured telephone interviews were conducted with parents of children who attended programs or clinics at 13 children's hospitals affiliated with FOCUS on a Fitter Future II (Table 1). Telephone interviews were conducted between June 2011 and September 2011. The joint institutional review board (IRB) of the University of Nebraska Medical Center/ Children's Hospital and Medical Center (Omaha, NE) was the IRB of record for five participating sites. Eight additional sites had independent approval by their own IRB. A 14th site had IRB approval to recruit patients, but did not enroll any patients in the study.

\section{Sample and Recruitment}

Parents/legal guardians of children who initiated participation in 13 stage 3 obesity programs/clinics between June 1, 2009 and February 1, 2011 were eligible to participate. Additional inclusion criteria included the following: (1) the patient did not complete $25 \%$ of the scheduled visits for programs with a defined number of visits; (2) the patient did not return for a follow-up clinic appointment within 6 months of his or her initial visit in a clinic without a defined number of visits; (3) the patient was not scheduled to return to the program/clinic (if applicable); (4) the patient was between 2 and 18 years of age at the time of program/clinic initiation; and (5) the parent was fluent in 
Table I. Participating Sites: Weight Management Format and Composition of Treatment

\begin{tabular}{|c|c|c|c|c|c|c|}
\hline \multirow[b]{2}{*}{ Site name } & \multicolumn{6}{|c|}{ Weight management treatment format and composition } \\
\hline & $\begin{array}{l}\text { Individual } \\
\text { visit }\end{array}$ & $\begin{array}{l}\text { Group } \\
\text { visits }\end{array}$ & $\begin{array}{c}\text { Individual-group } \\
\text { combined }\end{array}$ & Nutrition & $\begin{array}{l}\text { Behavioral } \\
\text { support }\end{array}$ & Medical \\
\hline Boston Children's Hospital (Boston, MA) & $x$ & & & $x$ & $x$ & $x$ \\
\hline $\begin{array}{l}\text { Children's Hospital and Medical Center } \\
\text { (Omaha, NE) }\end{array}$ & $x$ & $x$ & & $x$ & $x$ & $x$ \\
\hline $\begin{array}{l}\text { Children's Mercy Hospitals and Clinics } \\
\text { (Kansas City, MO) }\end{array}$ & $x$ & $x$ & $x$ & $x$ & $x$ & $x$ \\
\hline $\begin{array}{l}\text { Cincinnati Children's Hospital Medical } \\
\text { Center (Cincinnati, OH) }\end{array}$ & $x$ & $x$ & $x$ & $x$ & $x$ & $x$ \\
\hline CS Mott Children's Hospital (Ann Arbor, MI) & $x$ & $x$ & $x$ & $x$ & $x$ & $x$ \\
\hline Dell Children's Medical Center (Austin, TX) & $x$ & $x$ & $x$ & $x$ & $x$ & $x$ \\
\hline Florida Hospital for Children (Orlando, FL) & & & $x$ & $x$ & $x$ & $x$ \\
\hline $\begin{array}{l}\text { Mt. Washington Pediatric Hospital } \\
\text { (Baltimore, MD) }\end{array}$ & $x$ & $x$ & $x$ & $x$ & $x$ & $x$ \\
\hline $\begin{array}{l}\text { Nationwide Children's Hospital } \\
\text { (Columbus, OH) }\end{array}$ & $x$ & $x$ & $x$ & $x$ & $x$ & $x$ \\
\hline $\begin{array}{l}\text { New York Presbyterian Hospital-Weill Cornell } \\
\text { Komansky Center for Children's Health } \\
\text { (New York, NY) }\end{array}$ & & & $x$ & $x$ & $x$ & $x$ \\
\hline $\begin{array}{l}\text { Rainbow Babies and Children's Hospital } \\
\text { (Cleveland, OH) }\end{array}$ & $x$ & $x$ & $x$ & $x$ & $x$ & $x$ \\
\hline St. Louis Children's Hospital (St. Louis, MO) & & $x$ & & $x$ & $x$ & $x$ \\
\hline $\begin{array}{l}\text { University of Arkansas for Medical } \\
\text { Sciences/Arkansas Children's Hospital } \\
\text { (Little Rock, AR) }\end{array}$ & $x$ & $x$ & & $x$ & $x$ & $x$ \\
\hline
\end{tabular}

English or Spanish. Eligible participants were identified by study staff at each site, and telephone calls were made to recruit eligible parents. An optional cover letter explaining the study was sent to families in advance of telephone recruitment at some sites. If the parent was willing to participate in an interview, but was unable to complete the interview at the time of the phone call, the interview was scheduled for a subsequent time. Up to three attempts were made to contact parents to recruit them for this study. Informed verbal consent was obtained from parents before starting the telephone interview.

\section{Survey Instrument}

The semistructured interview was developed by two of the coauthors (E.T.R. and B.J.S.G.) and based on surveys administered at other sites, a review of the literature, and clinical experience. It was designed to assess for parent perspectives about what programs/clinics could do to retain families in weight management treatment. Openended questions were included to allow parents to describe (1) conversations their family had with programs/clinics about discontinuing, (2) what programs/clinics could have done to retain families, and (3) features of a program/clinic that would work best for their family. The interview also included a Likert scale rating of 48 different factors that may contribute to attrition. The open-ended questions and list of factors potentially associated with attrition were presented to the larger investigator group for review, and consensus was reached to finalize the survey. The survey was designed to be at a seventh-grade reading level. Results of the Likert scale ratings are presented elsewhere. ${ }^{30}$ The entire survey took approximately 10-20 minutes to administer. Likert scale ratings were administered first, followed by open-ended questions.

\section{Data Collection}

Because of differing IRB requirements and availability of equipment, not all institutions were able to audio record the interviews. For those institutions, detailed notes were taken instead. All interviewers were trained by the primary site investigator at their institution and were unknown to participants (i.e., nonclinical research staff). Interviews in Spanish were facilitated by Spanish translation of the survey provided by Dell Children's Medical Center (Austin, TX) and the use of bilingual interviewers, who recorded detailed notes in English. The child's height, weight, and demographic data, including race/ethnicity and insurance status, were abstracted from the clinical 
record. BMI-for-age percentile and $z$-score were calculated based on reference standards of the CDC. Survey data collected at each location were transferred to UH Rainbow Babies \& Children's Hospital (Cleveland, $\mathrm{OH}$ ) for data entry using TeleForm (Autonomy, San Francisco, CA), which was entered into a secure Microsoft Office database and stored on a secured SQL server. Demographic data were submitted to a central site for analysis at the Children's Hospital Association.

\section{Data Analysis}

The interview notes for the three qualitative questions were initially coded by two raters/authors per question to identify themes and preserve as much detail as possible. A coding guide with themes and subthemes was then developed for each question by the lead author (B.J.S.G.), who has experience with qualitative analysis. Table 2 displays the major themes for each qualitative question, as well as selected secondary subthemes. The questions were coded again

\section{Table 2. Summary of Major and Selected Secondary Coding Themes}

\section{Question}

Ia. Did you talk with members of the Did you talk with members of the decision to stop comir helpful?)

\section{Major and selected secondary themes}

- Positive staff interaction (affective quality of the relationship described as nonjudgmental, supportive, and/or kind)

- Staff provided education

- Staff offered return to program in the future

- Staff tried to address transportation needs

- Staff clarified reason for dropout

- Staff offered alternative location

- Parent felt better

Ib. Did you talk with members of the program/clinic about your decision to stop coming? (Why was that not helpful?)

- Time conflict

- No flexibility

- Program/clinic did not contact family

- Program/clinic dissatisfaction

- No follow-up to discuss concerns

- Staff encouraged family to give it more time

- Program/clinic unable to address problem

- Billing

- Transportation

2. Looking back on your experience with the program/ clinic, what could the program/clinic have done to keep you coming back?
- Program/clinic logistics

- More flexible hours

- More accessible treatment locations

- Number of staff

- Session length

- Frequency of visits

- Scheduling

- Copays

- Improvements for assessment/treatment planning

- Additional testing for causes of obesity

- Provide more specific recommendations

Provide "sneak peek" of program before entry

- Amount of information covered at the initial visit

- Improvements for treatment delivery

- Age appropriateness

- Increase contact with families

- Provide more treatment options

- Provide rewards

- Increase motivation

- Specific program assistance

- Transportation assistance

- Financial assistance

- Exercise resource assistance

- Improvements for staff-family interactions

- Increase parent encouragement

- Increase staff connection

- Increase staff sensitivity

- Increase cultural sensitivity

- Ensure family expectations are met

- Nothing

- Don't know 


\section{Table 2. Summary of Major and Selected Secondary Coding Themes continued}

\section{Question}

3. How would you describe a weight management program/ clinic that would work best for you and your child?
Major and selected secondary themes

- Program/clinic components

- Education

$\circ$ Group activities

Recommendations

- Program/clinic logistics

- Location

- Appointment times

- Frequency of appointments

- Program cost

- Characteristics of the clinic environment

- More selective admissions

Offer more treatment options

- Treatment delivery

- Type of visits

- Age-appropriate treatment delivery

- Format of program

- Family-staff interactions

- More interaction with staff

- Better staff connection with family

More effective communication

- Specific program/clinic assistance

- Transportation assistance

- Exercise resource assistance

- Provide rewards

- No suggestions by two raters/authors using the coding guide, and inter-rater reliability was calculated [i.e., number of agreements/ (number of agreements + number of disagreements)]. ${ }^{31}$ Interrater reliability was high for all questions (above 92\%). A third rater adjudicated any coding differences (B.J.S.G.). The results below are based on the consensus codes.

\section{Results}

A total of 13 children's hospitals participated, and 147 interviews were completed. The majority of parents interviewed were mothers $(87 \%)$. Children were mostly female $(56 \%)$, had a mean age of $10.8 \pm 3.3$ years, and mean BMI percentile of $99.2 \pm 1.9$. Approximately half were recipients of public insurance (48\%), and the group was racially diverse (36\% black, 35\% white, $6 \%$ Hispanic, $8 \%$ other, and $15 \%$ missing). Slightly more than half attended a clinic (56\%), compared to a program.

\section{Communication about Discontinuation}

Overall, in slightly more than half (53\%) of families, the parent alone made the decision to discontinue participation, whereas $8 \%$ of parents noted that their child made the decision. In $27 \%$ of the families, the decision was made jointly, involving the child in the decision. Only $3 \%$ of parents indicated that the family together with the program or clinic staff made the decision to discontinue participation. Responses from the remaining 9\% of families ranged from not being aware of follow-up appointments to only parents making the decision jointly with a staff member. All families were then asked whether they talked with members of the program/clinic about their decision to stop attending. The majority of parents $(65 \%)$ indicated that they did not talk to staff about their decision to discontinue treatment.

Among parents who did communicate with the program/ clinic about their decision to discontinue treatment, followup questions were asked to determine parental perception of the helpfulness of this discussion. For this study, "positive interactions" were coded if the parent described the affective quality of the relationship as nonjudgmental, supportive, and/or kind. More than half of parents with children in programs (i.e., program parents), who talked with their program, cited positive interactions with the staff as helpful (53\%), whereas no parents of children in clinics (i.e., clinic parents) made reference to positive interactions with staff. Instead, many clinic parents cited that discussions with staff were helpful because they received education $(43 \%)$ or were offered an opportunity to return in the future $(29 \%)$. Fewer program parents spontaneously cited these characteristics as helpful (education, 12\%; offer to return, 12\%). Typical responses included:

Program parent: They called me to follow up and I told them. They were nice, so I felt better.

Clinic parent: [They gave me] techniques to use at home and how to talk to [my] child about returning to the program.

Many clinic parents reported the discussion with staff was not helpful (64\%). When asked how the discussion was not helpful, clinic parents most frequently cited general 
clinic dissatisfaction (44\%), but also noted the clinic did not contact them to follow-up (22\%) or the clinic was unable to address a barrier to participation (22\%). For example:

Clinic parent: They just kept pushing us to come back.

Clinic parent: They could not adjust billing methods.

In contrast, program parents responded to this question by citing reasons for dropping out, such as time conflicts $(50 \%)$ and no program flexibility (33\%). A smaller percentage of program parents cited general program dissatisfaction (17\%). Examples include:

Program parent: They still didn't have different times.

Program parent: No, they did not offer any alternatives.

\section{Parental Suggestions for Better Patient Retention}

To inform how programs and clinics might improve retention rates, families were asked what changes they would recommend, which could have improved their retention. Both clinic and program parents most frequently discussed aspects related to changing program/clinic logistics (clinic parents, $33 \%$; program parents, $53 \%$ ) as the best means to improve retention. The most common responses from program parents in this category included a desire for more flexible hours (34\%) and more accessible treatment locations $(10 \%)$. The top two responses from clinic parents were similar, but to a lesser degree (flexible hours, $8 \%$; accessible treatment locations, $11 \%$ ). Less frequently cited responses related to logistics from both groups of parents included the scheduling process and copays. Typical responses about program/clinic logistics included:

Clinic parent: It was a train wreck. Appointments were at a bad time and inconvenient locations.

Program parent: Just the time. Something closer to 6:15 pm would be better. [Or] early morning on weekends.

Parents also cited improvements for treatment delivery (clinic parents, 18\%; program parents, 17\%) and improvements for assessment/treatment planning (clinic parents, $12 \%$; program parents, $9 \%$ ) as important factors in treatment retention. Many of these responses were related to tailoring treatment recommendations based on characteristics of the child. Some families (clinic parents, 6\%; program parents, 7\%) noted that specific assistance from the program/clinic, such as providing transportation, gas money, or help with obtaining healthy food, may have kept them coming back. Examples include:

Program parent: More follow-up from team members. Make sure the family got what they needed from the program.

Clinic parent: Offer more rewards and more options of ways to lose weight and reduce cholesterol.

\section{Tailoring the Treatment Options}

To better understand what families are looking for in pediatric weight management, they were asked to describe a program/clinic that would work best for them and their child.

When describing a program that would work best for their family, the most common theme among program parents was logistics (34\%), followed by the components of the program (27\%). Clinic parents also cited components (23\%) and logistics $(21 \%)$ most frequently. Many responses from both clinic and program parents relating to logistics pertained to more accessible locations and later or more convenient appointment times (e.g., after school, evening, weekend, or summer), although some parents noted a desire for a different frequency of appointments and lower program cost. Common responses included:

Program parent: It would've been helpful if it was offered on the weekend or in the summertime.

Clinic parent: Distance is a big factor, so something closer would be important.

Both clinic and program parents described a desire for components that are currently offered as part of standard weight management treatment, such as nutrition education (clinic parents, 12\%; program parents, 8\%), exercise (clinic parents, $7 \%$; program parents, $8 \%$ ), and behavior education/support (clinic parents, 4\%; program parents, $11 \%)$. Some parents discussed a desire for more specific and tailored dietary recommendations. For example:

Program parent: Focuses more on using types of food we already like. Portion control, using ingredients, and exercise.

Clinic parent: Personalized, one on one, deal with child and specific needs.

The third-most common theme was treatment delivery (clinic parents, 19\%; program parents, 16\%); however, there were no consistent responses within this theme for either program or clinic parents. For example, some parents discussed a desire for treatment to be delivered by individual sessions, whereas others expressed that group sessions would be helpful. Some parents noted ageappropriate treatment delivery and follow-up outside of regular sessions as important. Examples included:

Clinic parent: Individual counseling, group counseling, group activities with children of the same age going through similar problems.

Program parent: Full of activities. Fun place for them to work out and enjoy themselves so they'll want to come and lose weight and exercise. No pressure to lose weight, but at the same time they're losing weight.

More clinic (18\%) than program (3\%) parents described having a program/clinic offer financial assistance with transportation, exercise, and/or rewards. For example:

Clinic parent: One that didn't require child to miss so much school, memberships to YMCA activities.

Clinic parent: Free or low cost.

Program parent: Program that includes Tae Bo, Pilates, and yoga. Program provides transportation. 
A minority of families offered no suggestions (clinic parents, $10 \%$; program parents, $11 \%$ ) for a weightmanagement clinic or program that would work best for their family.

\section{Discussion}

Several studies suggest that sociodemographic characteristics, such as race/ethnicity, insurance, income, and education, are associated with attrition from pediatric weight management treatment. ${ }^{18,20,21,25,32}$ In this analysis, we sought to identify potentially modifiable aspects of patient attrition by soliciting input from families about their communication about treatment discontinuation, recommendations for better patient retention, and how clinics and programs could best meet families' goals. Overall, we found that most families discontinuing treatment report doing so on their own, without discussion of the decision with a member of their treatment team. However, among those who did discuss this decision with their treatment team, just over half found the discussion to be helpful. Logistical issues, such as flexibility in hours and location, were a central theme for families as both a factor that could affect their decision to return and that affected their view of how a pediatric weight management program or clinic could be adapted to best suit their needs.

Regardless of the type of treatment being offered, families expressed frustration about the limited flexibility in costs or hours and locations of the services offered. Similar issues have been noted in earlier studies. ${ }^{18,25-27}$ Unfortunately, some programs and clinics may have a limited ability to modify schedules or locations of services. The cost of services may also not be uniform because it may be linked to a family's health insurance and therefore also offers little opportunity for programmatic improvement.

Beyond logistical issues, when families were asked to describe a program/clinic that would work best for them, the components mentioned were often those already traditionally included in the approach to multidisciplinary pediatric weight management, such as nutrition education, exercise, and behavior education or support. Tailored treatments were also suggested as a factor that could influence a family's decision to return. It is possible that tailoring the main components of multidisciplinary weight management treatment to the specific family would be beneficial and enhance retention. Together, these comments suggest that in addition to the content, the method and/or frequency of delivery of specific services in pediatric weight management may need to be more individualized for each patient and family. For example, individual consultations may need to be offered for patients in group programs, and groups may need to be offered for patients in clinics with individual counseling. These comments are in keeping with earlier surveys of parents' reasons for dropout of pediatric weight management that have demonstrated roughly one third to one half of families reporting unmet expectations in their decision not to re- turn. ${ }^{18,25,26,28,29}$ Similarly, answers to the closed-ended Likert scale ratings in this survey also revealed implementation barriers, mismatched expectations, and motivation as important influences on their decision not to return. ${ }^{30}$ Because increasing attention is paid to the role of patient centeredness in the provision of healthcare, offering a range of clinical options for families as well as offering more information in advance about what to expect from the clinical experience may be opportunities for improvement that address these issues.

This study is one of only a few to specifically solicit input from parents regarding their views on attrition from pediatric weight management programs/clinics. It may be possible that parents chose to provide the least "offensive" answers and that possibly there were more personal views that they did not feel comfortable sharing. Nonetheless, to our knowledge, this study is the first to document parental experience of communication with the treatment team about the decision to discontinue the treatment as well as their experience about the usefulness of this discussion. Sampling from 13 different institutions provided both geographic diversity and the opportunity to evaluate differences experienced by families in clinics and programs. However, because not all sites were able to audio record the interviews, some responses were documented through detailed written notes. Although we do not believe that this influenced the gross scope or classification of the data, we are unable to determine whether additional minor categories would have been identified. In addition, respondents were parents of patients ranging from 2 to 18 years of age, chosen to evaluate the effect of differences in age on the factors related to attrition. Although our previous analyses revealed no effect of age on these factors, ${ }^{30}$ we cannot be certain that age would not affect the open-ended responses regarding experience about discontinuing treatment. Similarly, it is unknown if administering the Likert scale questions before the open-ended questions may have primed the parental responses. Last, families whose telephones were disconnected may have been underrepresented.

\section{Conclusion}

This study adds to the budding literature on patient attrition in pediatric weight management. Our findings suggest that discussing the decision to discontinue treatment for pediatric weight management may be an opportunity for re-engagement. Some families, who are seemingly lost to attrition, report that they were simply waiting to be contacted again. Others report that a conversation about their plans to discontinue treatment could have been helpful, in that greater tailoring of care might have kept them in a treatment setting that was otherwise not well matched to their current needs. Further research in this area should focus on improving communication, access to treatment and financial resources, as well as individualizing treatment based on families' needs and preferences. 
Given the chronicity of pediatric obesity, identifying reliable means of retaining families in programs and clinics for weight management must continue to be a critical focus of the providers and developers of these treatments.

\section{Acknowledgments}

The authors thank Stacy Biddinger, MPA (Children's Hospital Association), Michelle Demeule, MS, RD, LDN (Mt. Washington Pediatric Hospital), Kimberly Avila Edwards, MD (Dell Children's Medical Center), Ihouma Eneli, MD, MS (Nationwide Children's Hospital), Angela Fals, MD (Florida Hospital for Children), Mary Jane Hawkins, BSN, RN, CPN (Children's Hospital and Medical Center, Omaha), Shelley Kirk, PhD, RD, LD (Cincinnati Children's Hospital Medical Center), Nicole Kozma (St. Louis Children's Hospital), Patricia Morris, MPH (Children's Hospital Association), Amy Schmidt (UH Rainbow Babies and Children's Hospital), and Wendy Ward, PhD (Arkansas Children's Hospital), for their assistance with the project.

\section{Author Disclosure Statement}

The NACHRI (now the Children's Hospital Association) was the study sponsor. The study sponsor had no role in study design, collection of data, or the decision to submit the manuscript for publication. An editor supplied by the sponsor reviewed the final draft of the manuscript and a statistician conducted the demographic analyses. The sponsor hosted study-group meetings. The views expressed are those of the authors and do not necessarily reflect those of the Children's Hospital Association. Dr. Rhodes reports that she receives salary support from a philanthropic grant from the New Balance Foundation through the New Balance Foundation Obesity Prevention Center Boston Children's Hospital. No competing financial interests exist for the remaining authors.

\section{References}

1. Ogden CL, Carroll MD, Kit B, et al. Prevalence of obesity and trends in body mass index among US children and adolescents, 1999-2010. JAMA 2012;307:483-490.

2. Ogden CL, Carroll MD, Curtin LR, et al. Prevalence of high body mass index in US children and adolescents, 2007-2008. JAMA 2010;303:242-249.

3. Freedman DS, Dietz WH, Srinivasan SR, et al. The relation of overweight to cardiovascular risk factors among children and adolescents: The Bogalusa Heart Study. Pediatrics 1999;103:1175-1182.

4. Ogden CL, Yanovski SZ, Carroll MD, et al. The epidemiology of obesity. Gastroenterology 2007;132:2087-2102.

5. Schwimmer JB, Deutsch R, Kahen $\mathrm{T}$, et al. Prevalence of fatty liver in children and adolescents. Pediatrics 2006;118:1388-1393.

6. Sinha R, Fisch G, Teague B, et al. Prevalence of impaired glucose tolerance among children and adolescents with marked obesity. $\underline{N}$ Engl J Med 2002;346:802-810.
7. Wearing SC, Hennig EM, Byrne NM, et al. The impact of childhood obesity on musculoskeletal form. Obes Rev 2006;7:209-218.

8. Dietz WH. Health consequences of obesity in youth: Childhood predictors of adult disease. Pediatrics 1998;101:518-525.

9. Morrison JA, Glueck CJ, Horn PS, et al. Childhood predictors of adult type 2 diabetes at 9- and 26-year follow-ups. Arch Pediatr Adolesc Med 2010;164:53-60.

10. Let's Move Campaign. Available at www.letsmove.gov. Last accessed September 28, 2012.

11. Barlow SE; Expert Committee. Expert committee recommendations regarding the prevention, assessment, and treatment of child and adolescent overweight and obesity: Summary report. Pediatrics 2007;120(Suppl 4):S164-S192.

12. Hampl S, Paves H, Laubscher K, et al. Patient engagement and attrition in pediatric obesity clinics and programs: Results and recommendations. Pediatrics 2011;128(Suppl 2):S59-S64.

13. Whitlock EP, O'Connor EA, Williams SB, et al. Effectiveness of weight management interventions in children: A targeted systematic review for the USPSTF. Pediatrics 2010;125:e396-e418.

14. Faith MS, Van Horn L, Appel LJ, et al. Evaluating parents and adult caregivers as "agents of change" for treating obese children: Evidence for parent behavior change strategies and research gaps: A scientific statement from the American Heart Association. Circulation 2012;125:1186-1207.

15. Savoye M, Nowicka P, Shaw M, et al. Long-term results of an obesity program in an ethnically diverse pediatric population. Pediatrics 2011;127:402-410.

16. Skelton JA, Demattia LG, Flores G. A pediatric weight management program for high-risk populations: A preliminary analysis. Obesity 2008;16:1698-1701.

17. Skelton JA, Beech BM. Attrition in paediatric weight management: A review of the literature and new directions. Obes Rev 2011;12:e273-e281.

18. Cote MP, Byczkowski T, Kotagal U, et al. Service quality and attrition: An examination of a pediatric obesity program. Int J Qual Health Care 2004;16:165-173.

19. Kirk S, Scott BJ, Daniels SR. Pediatric obesity epidemic: Treatment options. J Am Diet Assoc 2005;105(5 Suppl 1):S44-S51.

20. Zeller M, Kirk S, Claytor R, et al. Predictors of attrition from a pediatric weight management program. J Pediatr 2004;144:466-470.

21. Dolinsky DH, Armstrong SC, Ostbye T. Predictors of attrition from a clinical pediatric obesity treatment program. Clin Pediatr (Phila) 2012;51:1168-1174.

22. Murtagh J, Dixey R, Rudolf M. A qualitative investigation into the levers and barriers to weight loss in children: Opinions of obese children. Arch Dis Child 2006;91:920-923.

23. Twiddy M, Wilson I, Bryant M, et al. Lessons learned from a family-focused weight management intervention for obese and overweight children. Public Health Nutr 2012;5:1-8.

24. Lindelof A, Nielsen CV, Pedersen BD. Obesity treatment-more than food and exercise: A qualitative study exploring obese adolescents' and their parents' views on the former's obesity. Int $J$ Qual Stud Health Well-being 2010;5:1-11.

25. Barlow SE, Ohlemeyer CL. Parent reasons for nonreturn to a pediatric weight management program. Clin Pediatr (Phila) 2006;45: 355-360.

26. Skelton JA, Goff DC, Jr., Ip E, et al. Attrition in a multidisciplinary pediatric weight management clinic. Child Obes 2011;7:185-193.

27. Kitscha CE, Brunet K, Farmer A, et al. Reasons for non-return to a pediatric weight management program. Can J Diet Pract Res 2009;70:89-94. 
28. Banks J, Cramer H, Sharp DJ, et al. Identifying families' reasons for engaging or not engaging with childhood obesity services: A qualitative study. J Child Health Care 2013 May 31. doi: 10.1177/ 1367493512473854. [Epub ahead of print]

29. Visram S, Hall TD, Geddes L. Getting the balance right: Qualitative evaluation of a holistic weight management intervention to address childhood obesity. J Public Health (Oxf) 2013;35:246-254.

30. Hampl S, Demeule M, Eneli I, et al. Parental perspectives on attrition from tertiary care pediatric weight management programs. Clin Pediatr (Phila) 2013;6:513-519.

31. Miles M, Huberman AM. Qualitative Data Analysis: An Expanded Sourcebook. Sage: Thousand Oaks, CA, 1994.

32. Tershakovec AM, Kuppler K. Ethnicity, insurance type, and follow-up in a pediatric weight management program. Obes Res $2003 ; 11: 17-20$.
Address correspondence to: Bethany J. Sallinen Gaffka, PhD Clinical Assistant Professor, Associate Director for Psychological Services Pediatric Comprehensive Weight Management Center Department of Pediatrics Division of Child Behavioral Health University of Michigan Health System 1500 East Medical Center Drive, SPC 5318 Ann Arbor, MI 48109-5318

E-mail: bsalline@med.umich.edu 
This article has been cited by:

1. Rhodes Erinn T. , MD, MPH , 1 Boles Richard E. , PhD , 2 Chin Kimberly , BA , 3 Christison Amy , MD , 4 Testa Elizabeth Getzoff, PhD , 5 Guion Kimberly, PhD , 6 Hawkins Mary Jane, BSN, RN, CPN , 7 Petty Carter R. , MA , 3 Sallinen Gaffka Bethany, PhD , 8 Santos Melissa , PhD , 9 Shaffer Laura, PhD , 10 Tucker Jared , PhD , 11 and Hampl Sarah E. , MD 12 1Division of Endocrinology, Boston Children's Hospital, Boston, MA. 2Children's Hospital Colorado, Aurora, CO. 3Clinical Research Center, Boston Children's Hospital, Boston, MA. 4Children's Hospital of Illinois, Peoria, IL. 5Mt. Washington Pediatric Hospital, Baltimore, MD. 6Oregon Health and Science University, Portland, OR. 7Children's Hospital and Medical Center, Omaha, NE. 8Pediatric Comprehensive Weight Management Center, University of Michigan Health System, Ann Arbor, MI. 9Connecticut Children's Medical Center, Hartford, CT. 10University of Virginia, Charlottesville, VA. 11Helen DeVos Children's Hospital, Grand Rapids, MI. 12Pediatrics and Center for Children's Healthy Lifestyles \& Nutrition, Children's Mercy Hospital, Kansas City, MO. . 2017. Expectations for Treatment in Pediatric Weight Management and Relationship to Attrition. Childhood Obesity 13:2, 120-127. [Abstract] [Full Text HTML] [Full Text PDF] [Full Text PDF with Links] [Supplemental Material] 